\title{
(Why Aren't We) \\ Solving Common Library Problems with Common Systems?
}

May Yan and MJ Suhonos

Ryerson University 


\section{$\mathrm{ERM}=$ Problem Solving}

- Book is listed in our catalogue but we've lost access on book platform

- Journal thresholds are differently listed between our catalogue, the link resolver, and the publisher platform - which is correct?

- When did we cancel Teaching Professor and why?

- We need to weed the print copies of periodicals in Chemical Engineering to make space, which ones do we have perpetual rights to online?

- Can you post a PDF of this SUPO book we have from Ebook Central on ARES? 


\section{Clues - ERM Records}

Records might be:

- License Agreements

- Invoices

- Titles Lists

- Kbart files

- Emails

- Meeting Agendas

For our own use but also evidence in our interactions with outside parties (vendors, publishers, suppliers) and legal obligation (legal discovery). 


\section{Need Records Management}

Step 1: Developed a Records Retention Schedule

[Records Retention Schedule: Define what to keep, for how long, and when and how to dispose of it/archive it.]

Step 2: Implement the Schedule 


\section{Management Records with System}

- University Records Management processes handles paper records

- No University wide electronic Records Management system

- Need electronic solution - don't want to wait

- Priority: need to preserve records that document evidence of legal rights to perpetual access resources (Archives where we have right to retain access on vendor platform, dark archives or manage a copy in case of cancellation or ceased publication, operation of business etc.)

- We have very limited resources - need low effort solutions 


\section{Functional Requirements}

Must integrate with current ERM workflow; easy to use; restrict users into system

Metadata: Publisher, Resource, File type, File Name, Duration, File Status, Date acquired, etc.

Search: By metadata; Full text searchable (PDF, excel, word, etc);

Accession/Preservation/Destruction based on file type, status and schedule with focus on Preservation and scheduled destruction. 


\section{AtoM - A Library System}

- Existing experience in Archives \& Special Collections

- Low-cost, easy to deploy

- Compatible data model (File, Record, Actor)

- Customizable (UI, taxonomies)

- Full-text indexing, faceted search

- Verdict: workable but development required 


\section{Strategic Systems Requirements Review}

Strategic Analysis to define requirements of Library's Collections Systems.

In Scope: ILS, ERM systems, IR, Discovery, Reserves, ILL etc.

Not in Scope: Research Guides, Reference, Instruction etc.

Methodology used: Task Descriptions (Lauesen, 2003)

This approach allows us to define our problems and then search for technology solutions. 


\section{Subset of Strategic Recommendations}

1. Software as a service (SaaS) model

2. Choose the best of breed product for our needs and swap out for better technology solutions as often as possible.

4. use open source technologies for our needs when appropriate.

5. Where there is no significant advantage to a library service provider solution, use products outside of the library technology 


\section{Strategic Goals}

- Modularity to maximize flexibility

- Consistency across applications

- Control feature/requirements scope

- Abstract implementation from service

- Reduce maintenance \& security risks

- Commodity solutions when possible 


\section{Common Problems}
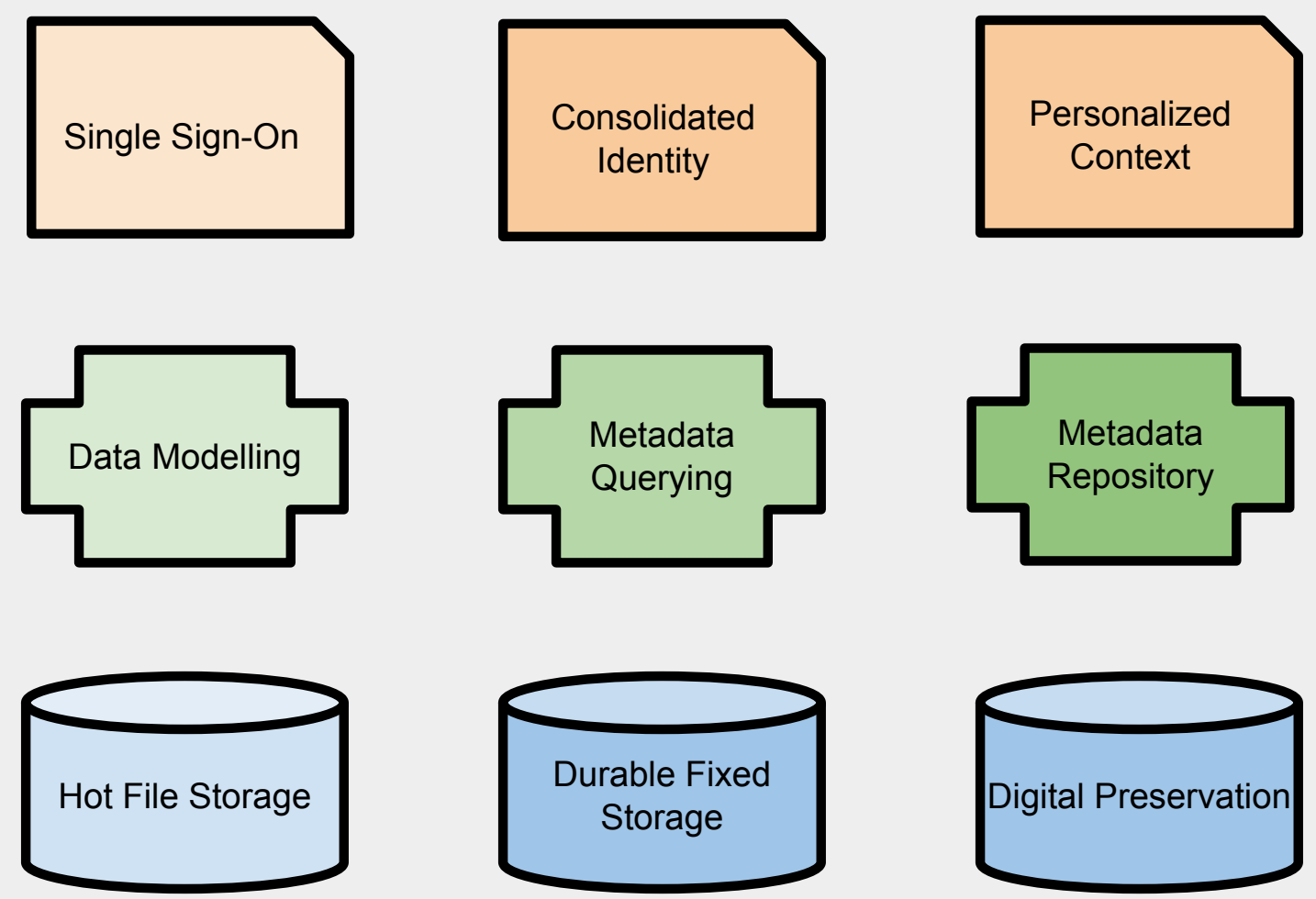


\section{Single Sign-On}
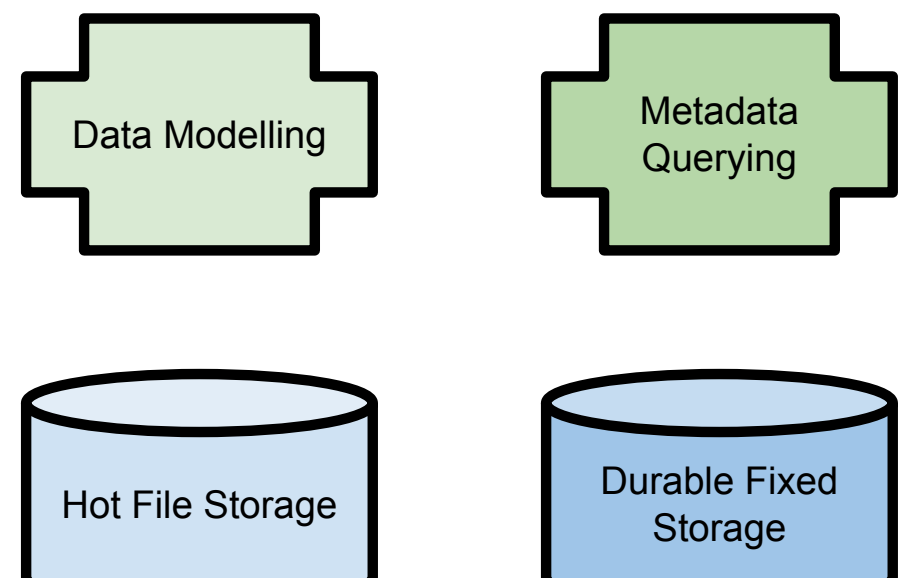
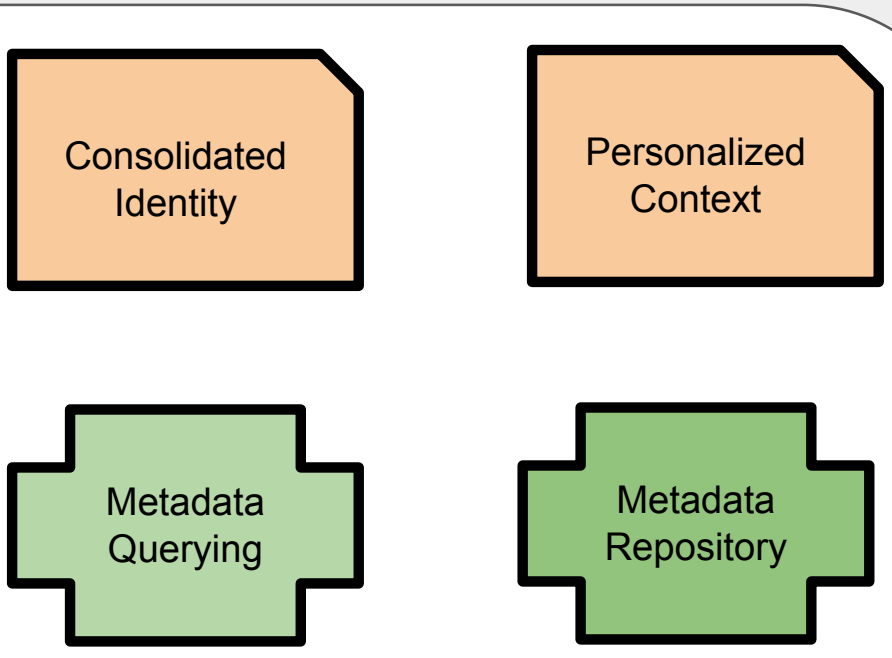

Durable Fixed Storage

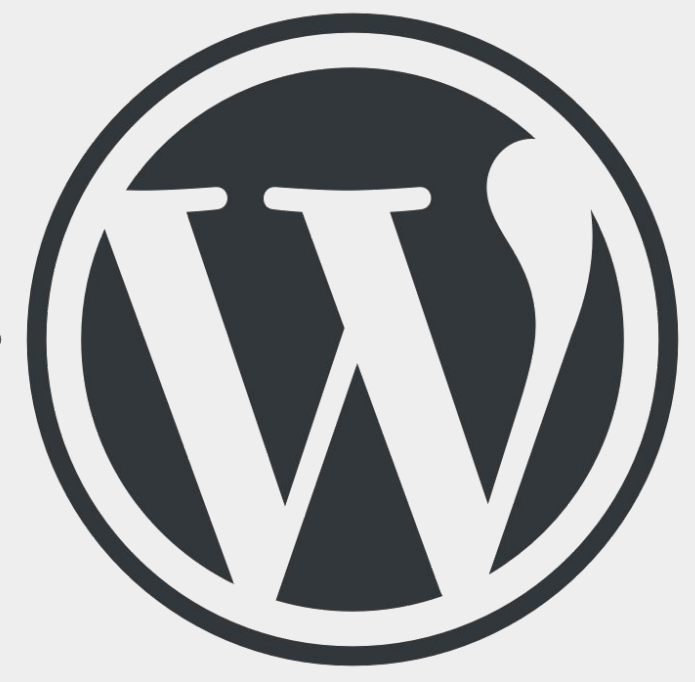




\section{Wordpress - A Common System}

- Open source, well-established CMS

- Existing general familiarity among all staff

- Flexibility for future development and customization

- Knowledge becomes a common part of the Library

- Facilitates working with research partners

- Mature SaaS options and plug-ins available

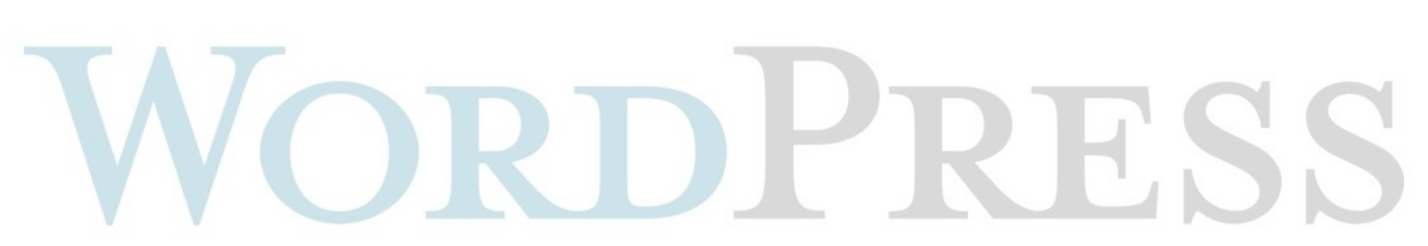




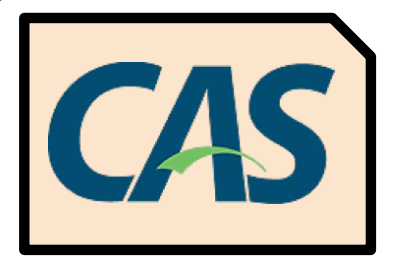

Pods ectous

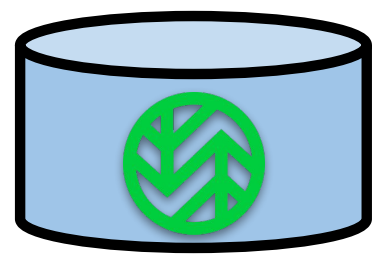

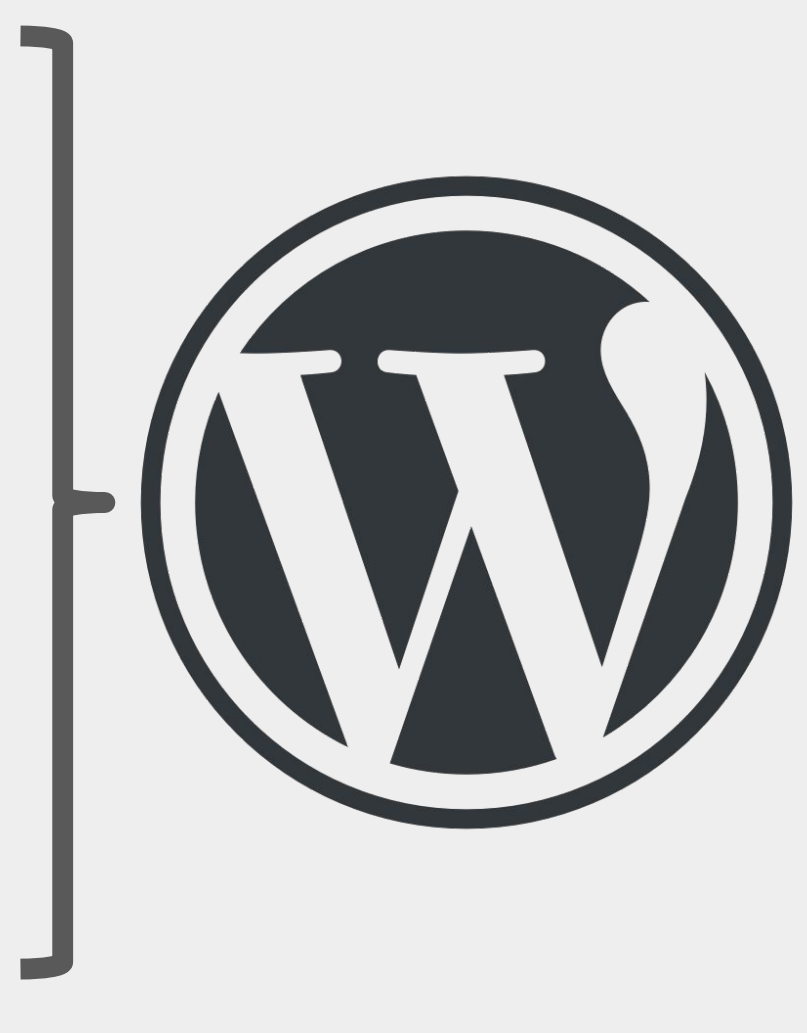


Filters

$\square$ All Results

\section{Type}

$\square$ Actor (9)

$\square$ Document (5)

Actor type

$\square$ Corporation (3)

$\square$ Person (3)

$\square$ Consortium (1)

$\square$ Nonprofit (1)

$\square$ University (1)

Document type

$\square$ License Agreement (5)

\section{Document status}

$\square$ Inactive (3)

$\square$ Active (2)

Resource

Cambridge Journals Online $\square$ (2)

\section{Cambridge University Press Journals License}

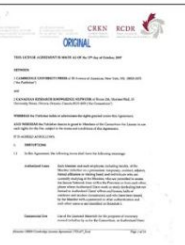

\section{Canadian Research Knowledge Network}

\author{
Jim MacLeod
}

BBM Canada 


\section{All Good}

\section{Freedom to do this}

- Scope

- Low risk application (internal)

- Planning required to execute

- Internal culture

- Okay to fail

- Can develop and operate outside of University IT oversight 


\section{Potential Applications}

- Institutional repository

- Archival collections system

- Catalogue / OPAC

- Departmental intranet

- Faculty research projects 


\section{Contact Us}

may.yan@ryerson.ca

@mayyan

mjsuhonos@ryerson.ca

\section{Discussion}

Questions? 